\title{
Characterization of pressure dynamics in an axisymmetric separating/reattaching flow using fast-responding pressure-sensitive paint
}

\author{
Martin Bitter · Tatsuya Hara • Rainer Hain • \\ Daisuke Yorita $\cdot$ Keisuke Asai $\cdot$ Christian J. Kähler
}

Received: 27 April 2012/Revised: 24 August 2012/Accepted: 28 August 2012/Published online: 4 October 2012

(C) The Author(s) 2012. This article is published with open access at Springerlink.com

\begin{abstract}
This collaborative work discusses the results of time-resolved pressure-sensitive paint measurements performed on a model of a generic spacecraft under sub- and transonic test conditions. It is shown that optical pressure measurements using an active layer from platinumporphyrin complexes (PtTFPP) in combination with a polymer-ceramic base layer are able to measure dynamic flow phenomena in the trisonic wind tunnel facility up to sampling rates of $2 \mathrm{kHz}$. Low amplitude fluctuations in the order of $0.1 \mathrm{kPa}$ were determined by means of this measurement technique. The buffet dynamics, as well as the spatial extent of the recirculation area in the near-wake, compare well with numerical predictions and PIV measurements. Furthermore, characteristic coherent pressure modes on the base were resolved, which were predicted by large-eddy simulations.
\end{abstract}

\section{Introduction}

A joint research project was initiated for the development of economic and safe concepts for future space missions, which is entitled: Technological foundations for the design

\footnotetext{
M. Bitter $(\varangle) \cdot$ R. Hain · C. J. Kähler

Institute of Fluid Mechanics and Aerodynamics,

Bundeswehr University Munich, Werner-Heisenberg-Weg 39,

85577 Neubiberg, Germany

e-mail: martin.bitter@unibw.de

C. J. Kähler

e-mail: christian.kaehler@unibw.de

T. Hara $\cdot$ D. Yorita $\cdot$ K. Asai

Department of Aerospace Engineering, School of Engineering,

Tohoku University, 6-6-01 Aramaki- Aza-Aoba Aobaku,

Sendai 980-8579, Japan
}

of thermally and mechanically highly loaded components of future space transportation systems. The project is funded by the Deutsche Forschungsgemeinschaft (DFG). Within this project, the Institute of Fluid Mechanics and Aerodynamics of the Bundeswehr University in Munich (UniBwM) is fundamentally interested in the characterization of the dynamic loads caused by the fluid-mechanical interaction between the flow and the structure of the nozzle shortly after take-off $\left(\mathrm{M}_{\infty}=0.3\right)$ and at transonic conditions $\left(\mathrm{M}_{\infty}=0.7\right)$. During these conditions, largescale vortex structures detach from the base of the spacecraft and interact with its main exhaust nozzle. This leads to high mechanical loads and, moreover, raises critical safety aspects (Gülhan 2008).

Detailed flow investigations based on PIV measurements are outlined in Bitter et al. (2011) and Scharnowski and Kähler (2011), which indicate the topology and dynamics of the wake. These results are in good agreement with the numerical investigations by Statnikov et al. (2012). However, the numerical prediction of a mode-like structure on the base of the configuration could not be validated experimentally so far. This is one scope of this article. In addition, the results are analyzed to answer the following scientific questions: (1) How is the dynamic behavior of the coherent wake structures characterized? (2) Are the dynamic phenomena dominated by a certain frequency? (3) Does the boundary layer/wake interaction result in a coherent mode pattern on the base? (4) Is this mode pattern somehow timedependent? The identification of convecting structures, especially the stream-wise convection behind a $2 \mathrm{~d}$ or axisymmetric backward-facing step, was extensively studied in the literature for a large variety of flow properties, see Eaton (1980), Lee and Sung (2001, 2002), Spazzini et al. (2001) and Mabey (1972). To answer the questions mentioned above, the time-resolved pressure-sensitive paint 
measurement technique is required. The physical working principle of the pressure-sensitive paint (PSP) measurement technique is based on the detection of fluorescence intensities. Oxygen-reactant molecules (e.g. porphyrin or pyrene) are excited to an unstable higher energy state by short-wave radiation (near UV). The molecules can emit long-wave radiation during their recurrence into the stable ground state. This radiation can be registered with a photoelectric cell. The intensity of the delivered radiation is dependent on the oxygen concentration in the closer surroundings of the excited molecule. The excited molecule will likely collide with an oxygen molecule and lose its energy due to this interaction, if the $\mathrm{O}_{2}$-concentration is high. This incident is known as oxygen-quenching. Quenched molecules relapse back into the ground state without radiation. The $\mathrm{O}_{2}$-concentration is directly proportional to the oxygen partial pressure and, therefore, to the static pressure of the fluid, according to Henry's law. The behavior of a luminophor quenched by oxygen molecules can be described by the Stern-Volmer relation (1):

$I_{0} / I=1+c_{\mathrm{q}}(T) \cdot p_{\mathrm{O}_{2}}$

where $I_{0}$ is the luminescence intensity at vacuum conditions, $I$ the detected luminescence intensity, $c_{\mathrm{q}}(T)$ the temperature-dependent quenching constant and $p_{\mathrm{O} 2}$ the oxygen partial pressure. The Stern-Volmer relation is slightly modified for its use in PSP applications to relation (2):

$p / p_{\text {ref }}=c_{1}(T)+c_{2}(T)\left(I_{\text {ref }} / I\right)+\cdots+c_{n}(T)\left(I_{\text {ref }} / I\right)^{n}$

for the determination of unknown static surface pressures $p$. Relation (2) is commonly used up to a second-order formulation. It contains temperature-dependent calibration coefficients $c_{n}(T)$, which are luminophore-specific parameters and which can be determined in a static calibration chamber. Furthermore, it contains the measured luminescence intensity $I_{\text {ref }}$ and static pressure $p_{\text {ref }}$ at a reference state (known as wind-off condition) and the measured luminescence intensity $I$ under flow conditions (known as wind-on condition). Hence, the static pressure $p$ can be calculated by determining the paint characteristic coefficients and the intensity ratio $I_{\text {ref }} / I$. More thorough explanations of this measurement technology can be found in Liu and Sullivan (2005).

The worldwide application of PSP to assess fluidmechanical problems in wind tunnels at larger research facilities like NASA, DLR, JAXA, ONERA dates back to the mid-1990s. As reported by Airaghi (2006), Crafton et al. (1999), Nakakita (2007), Woodmansee and Dutton (1998), Yang et al. (2012), this technique is increasingly used at university departments. The Institute of Fluid Mechanics and Aerodynamics began its installation of a PSP-system several years ago, see Bitter et al. (2009).
An overview of recent approaches for the optical determination of unsteady surface pressure distributions with instationary pressure-sensitive paint (iPSP) can be found in Gregory et al. (2008).

Currently, time-varying processes with characteristic frequencies up to $20 \mathrm{kHz}$ can be resolved precisely with this technique, according to Gregory et al. (2007). To achieve short-duration response times within a few microseconds, the anchoring of the PSP-molecules, directly at the surface of the pursued object, is desired to preserve the shortest possible diffusion time between the oxygen molecules and the PSP dye. An anchoring of the molecules in a porous sponge-like surface structure is desired in order to ensure that the molecules stick to the surface and do not get removed immediately by high shear forces under transonic test conditions. Two ways of creating a porous surface structure have been established for unsteady PSP measurements over the years. On the one hand, a porous surface structure is created by anodizing the model surface by an electro-chemical catalysis process. This technique was first developed by Asai et al. (1998) and was further improved to resolve flow phenomena with large dynamic ranges, see Gregory et al. (2007), Kameda et al. (2005), Mérienne et al. (2004), Sakaue et al. (2006), Singh et al. (2011). A second approach is the application of an emulsion to the model surface, which becomes a highly porous structure after drying, see Asai et al. (2001), Juliano et al. (2012), Klein et al. (2010). In both cases, the active polymer is later anchored directly into the porous structure either by dipping or by spraying.

\section{Experimental setup and methodology}

\subsection{Fast-responding pressure-sensitive paint}

A porous carrying layer based on a polymer-ceramic compound (pc-PSP) dissolved in distilled water was used for the investigations presented here. The formulation for this compound was introduced in Gregory et al. (2007). For every $1.2 \mathrm{~g}$ of distilled water, $1.73 \mathrm{~g}$ of titanium dioxide and $12 \mathrm{mg}$ of a dispersant, Duramax D 3005, were mixed together and stirred for about $1 \mathrm{~h}$ to generate the compound. Afterward, the mixture was combined with 3.5 vol- $\%$ of a ceramic emulsion, Rhoplex RH8, and stirred again for several minutes. A modification in the amount of distilled water has been made due to slightly larger titanium dioxide particles compared to the originally proposed formulation in Gregory et al. (2007) ending up in a much smoother surface with the same porosity. The final mixture was applied to the model by spray gun. A base-layer thickness of about 15 microns was obtained. Platinum-porphyrin complexes (PtTFPP) were used as pressure-sensitive molecules 


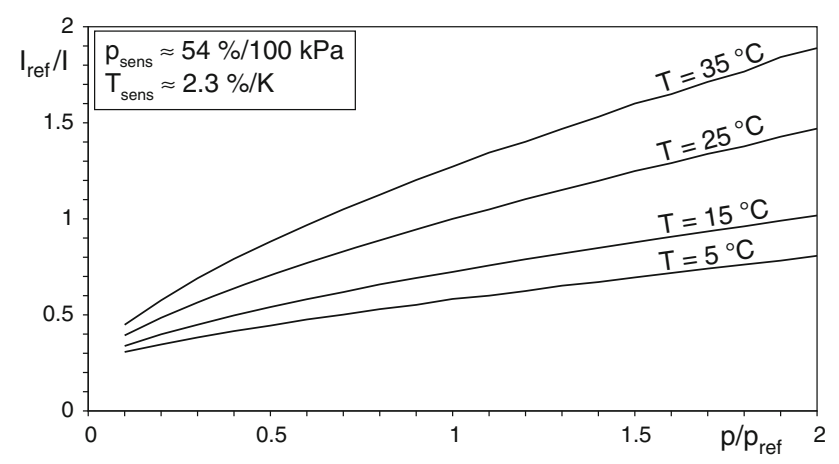

Fig. 1 Stern-Volmer calibration plots for instationary pressure-sensitive paint on a polymer-ceramic base layer (pc-PSP) normalized at reference conditions $p_{\text {ref }}=100 \mathrm{kPa}$ and $T_{\text {ref }}=25^{\circ} \mathrm{C}$

in a single component PSP using a solution of $4 \mathrm{mg}$ PtTFPP dissolved in $20 \mathrm{ml}$ of toluene. This composition was also applied by spray gun. The pc-PSP paint properties, which were qualified in a static calibration chamber, are presented in Fig. 1. The pressure sensitivity was determined to be $p_{\text {sens }} \approx 54 \% / 100 \mathrm{kPa}$ and the temperature dependence was $T_{\text {sens }} \approx 2.3 \% / \mathrm{K}$. Due to the high temperature dependence, the knowledge of the surface temperature at the wind-on and wind-off condition is essential. A RANS-CFD solution, performed by the authors at $\mathrm{M}_{\infty}=0.7$ conditions, offered information regarding the surface temperature gradient. A maximum temperature change of $\Delta T \approx 2 \mathrm{~K}$ was found in the desired region-of-interest. In combination with the temperature sensitivity of pc-PSP, an in-situ pressure error of about $5 \%$ is expected by the temperature sensitivity.

The first-order transfer function of pc-PSP was evaluated in a high-frequency acoustic tube within a frequency range of $0.1 \leq f \leq 10 \mathrm{kHz}$ by Sugimoto et al. (2012). As reported, no significant amplitude drops (|Gain| $\leq 1 \mathrm{~dB}$ ) or phase lags (IPhasel $\leq 10^{\circ}$ ) occur for pc-PSP up to sampling rates of $f \approx 2 \mathrm{kHz}$. Hence, no amplitude or phase correction was applied to the results from these experiments.
Figure 2 shows the model coated with iPSP for the individual test series. Adjacent surfaces that are not of interest were covered with diffuse black paint for the suppression of intensity superposition, called self-illumination. Self-illumination might cause errors in the intensity ratio in the order of several percent, according to Ruyten (1997) or Le Sant (2001). This error was almost totally suppressed by the black paint.

\subsection{Spacecraft model for unsteady pressure measurements}

The geometry of the generic spacecraft model was presented in detail in previous works, see Bitter et al. (2011) or Scharnowski and Kähler (2011). A sketch of the model, which was specifically developed for unsteady pressure measurements, is shown in Fig. 3. The model was designed in a shell-like construction to allow for the equipment with static and unsteady pressure and temperature sensors. There are 4 static pressure ports $\left(p_{\mathrm{s}}\right.$ in Fig. 3 ) on the cylindrical part, circumferentially distributed over $90^{\circ}$, which were used to avoid a potentially oblique installation of the model. Furthermore, the model includes 4 static temperature sensors; 2 at the cylindrical part and 2 inside the base ( $T$ in Fig. 3). These were incorporated into the model's surface by a thermal adhesive. The base is equipped with 13 unsteady pressure transducers ( $p$ in Fig. 3), whereas only 4 sensors could be read out simultaneously at the time of these experiments. The suspension of the model in the test section was realized via a rear-sting mounting. The diameter of the sting holds the scaled nozzle diameter, which is placed on the original equivalent instead. The length of the nozzle would be about 1.2 times the model diameter. The sting mount carried no temperature and pressure sensors for manufacturing reasons. A temperature sensor at the base was used for the correction of the PSP-temperature effect on the sting, assuming that the sting experiences the same temperature change during a

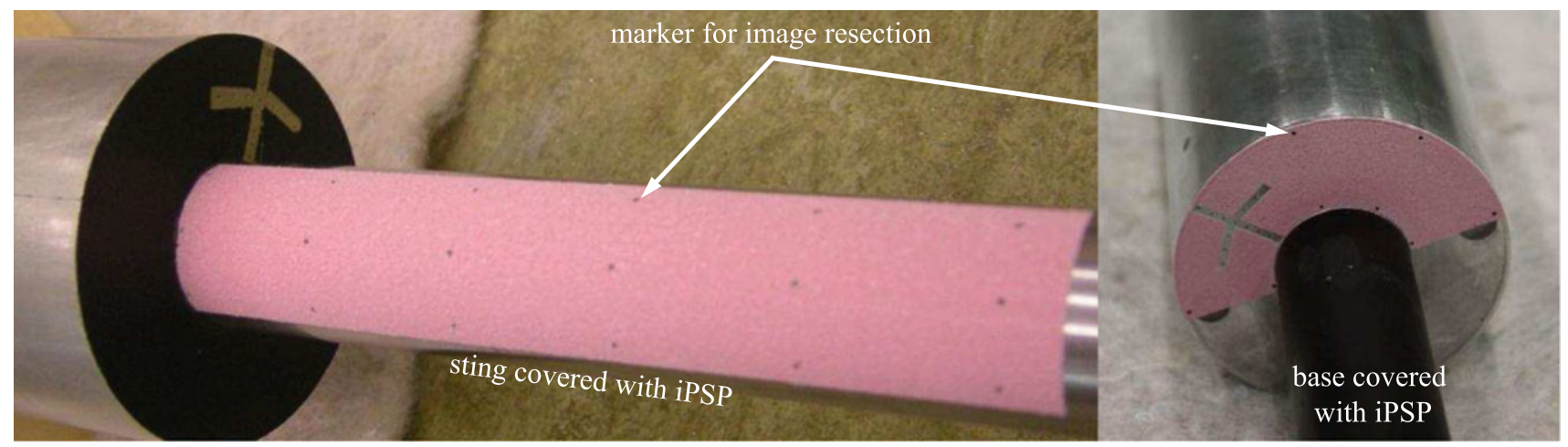

Fig. 2 Left rear-sting mounting covered with pc-PSP for wake dynamics characterization; Right base covered with iPSP for mode structure investigations 


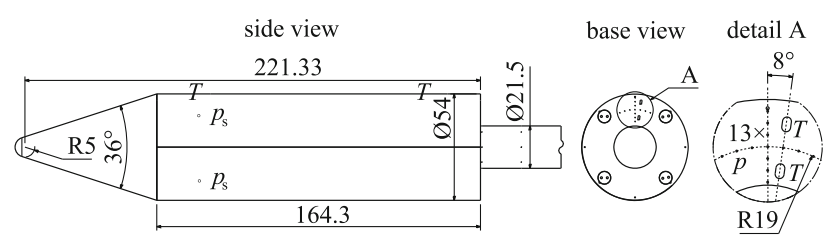

Fig. 3 Modular generic space transporter model made from aluminum. Left full view on the model including rear-sting support for model mounting; Right view on the model base. The model is equipped with 13 unsteady pressure transducers $\left(p_{\mathrm{s}}\right), 4$ static pressure ports $(p)$ and 4 static temperature sensors $(T)$

wind tunnel run as the base. The rear-sting mount was used to ensure symmetric flow conditions. The disadvantage of this type of model suspension is a potential delay (or even the complete prevention) of the reattachment of the re-circulation domain in the wake. However, as examined in Bitter et al. (2011) and Scharnowski and Kähler (2011), this effect only occurs for inlet Mach numbers of $\mathrm{M}_{\infty}>2$ and is no problem at subsonic conditions.

\subsection{Wind tunnel setup}

The experiments were split into two test series in order to answer the scientific key questions. Regarding the dynamic interaction of large-scale flow structures with the nozzle (rear-sting mounting), the sting was coated with pc-PSP within a range of $100 \mathrm{~mm}$ or about 1.8 diameters downstream of the model base, see left side of Fig. 2. The base was circumferentially coated with pc-PSP over $180^{\circ}$ for the second test series (right side in Fig. 2) in order to resolve a possible characteristic distribution of pressure fluctuations.

The experiments were carried out in the Trisonic Wind tunnel Munich (TWM). This blow-down type wind tunnel with cross-section dimensions of $0.68 \times 0.3 \mathrm{~m}^{2}$ and a maximum operating time of $350 \mathrm{~s}$ at $\mathrm{M}_{\infty}=3$ is driven by compressed air, which is expanded out of its storage reservoirs from $p_{\mathrm{t}}=20$ bar down to operating total pressures in the range of $p_{\mathrm{t}}=[1, \ldots, 5]$ bar. By this variation, the Reynolds number can be set between $R e=[7, \ldots, 80] \times$ $10^{6} \mathrm{~m}^{-1}$. The Mach number range is between $\mathrm{M}_{\infty}=$ $0.3, \ldots, 3.0$. The turbulence level at subsonic conditions is around $T u \approx 1.2 \%$. Two setups, shown schematically in Fig. 4, were subsequently installed at the wind tunnel.

A Phantom V.12 high-speed CMOS camera (a) with a sensor size of $1 \mathrm{Mpx}$ and a frame rate up to 6,200 frames/s at full frame size was used for both test series. The camera was installed out of the plenum chamber at a distance of about $1.4 \mathrm{~m}$ away from the PSP surface in setup 1 . In the second setup, the camera was attached directly to the wind tunnel inside the plenum chamber at a distance of about 0.4 $\mathrm{m}$. A high pass filter with a cut-on wavelength of $570 \mathrm{~nm}$, $T \geq 93 \%$ transmission and an optical density OD $>6$ was

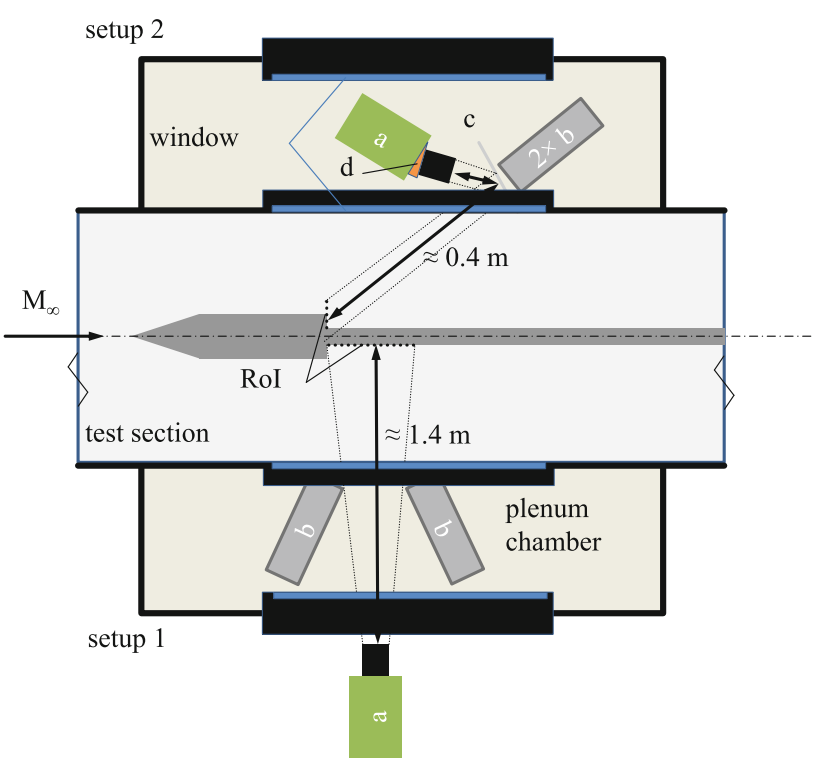

Fig. 4 Non-scaled schematic of the experimental setups for the nearwake characterization (setup 1) and for the base flow investigations (setup 2) with camera (a), excitation LED (b), mirror $(c)$ and Scheimpflug-angle correction device $(d)$

used in front of the objective lens in order to separate the luminescence signal from the excitation. Two Luminus $C B V-120 U V$ high power LEDs (b), each with $10 \mathrm{~W}$ optical power, were operated in continuous-wave (c-w) mode for the excitation of the PSP. A spherical lens with $f=$ $-75 \mathrm{~mm}$ focal length and a diameter of $d=120 \mathrm{~mm}$ maximizes the excitation intensity from the LEDs for short integration times and ensures a homogeneous light distribution. The LEDs were installed directly on the window of the test section in both setups. The camera view was deflected with a mirror (c) in setup 2 to allow for the observation of the base area from the back of the model. An adjustment of the depth of focus using a Scheimpflug device (d) accounted for the oblique viewing plane. The specific test conditions and recording parameters of both series of experiments are given in Table 1.

\subsection{Methodology}

\subsubsection{Image acquisition}

The wind tunnel run time, including the control process, was about $60 \mathrm{~s}$ at its maximum during transonic test conditions. The flow temperature and hence the temperature of the model surface changes strongly during the individual experiments due to the expansion of compressed air from the storage vessels into the wind tunnel. Thus, a stable surface temperature distribution could not be realized. Because of the temperature sensitivity of pc-PSP, the measurement of the surface temperature is essential. 
Table 1 Flow conditions and acquisition parameter for the iPSP measurements

\begin{tabular}{|c|c|c|c|c|c|}
\hline \multirow{2}{*}{$\frac{\text { Region-of-interest RoI }}{\text { Mach number } \mathrm{M}_{\infty}}$} & \multicolumn{3}{|c|}{ Sting } & \multicolumn{2}{|c|}{ Base } \\
\hline & & & $0.3 ; 0.7$ & & \\
\hline Reynolds number $\operatorname{Re}_{\mathrm{D}}[\cdot 106]$ & & & $0.65 ; 1.0$ & & \\
\hline Run total pressure $p_{\mathrm{t}}[\mathrm{kPa}]$ & & & $170 ; 150$ & & \\
\hline Run static pressure $p_{\mathrm{s}}[\mathrm{kPa}]$ & & & $160 ; 107$ & & \\
\hline iPSP sampling rate $f_{\mathrm{s}, \mathrm{iPSP}}[\mathrm{kHz}]$ & 1 & 2 & 4 & 1 & 2 \\
\hline iPSP cutoff frequency $f_{\mathrm{c}}[\mathrm{Hz}]$ & 512 & 1,024 & 2,048 & 512 & 1,024 \\
\hline iPSP integration time $t_{\mathrm{i}, \mathrm{PPSP}}[\mu \mathrm{s}]$ & 999 & 499 & 249 & 999 & 499 \\
\hline kulite sampling rate $f_{\mathrm{s}, \mathrm{kul}}[\mathrm{kHz}]$ & & & 10 & & \\
\hline Optics & \multicolumn{3}{|c|}{ Zeiss Sonnar T 180 mm, F5.6 } & \multicolumn{2}{|c|}{ Zeiss Macro-Planar T* $50 \mathrm{~mm}, \mathrm{~F} 4$} \\
\hline Field-of-view $W \times H\left[\mathrm{~mm}^{2}\right]$ & $106.4 \times 32.2$ & $80.5 \times 23.4$ & $51.8 \times 17.6$ & $31.4 \times 57.5$ & $31.4 \times 57.5$ \\
\hline Pixel array $\left[\mathrm{px}^{2}\right]$ & $1184 \times 352$ & $896 \times 256$ & $576 \times 192$ & $336 \times 768$ & $336 \times 768$ \\
\hline Magnification m & \multicolumn{3}{|c|}{$1: 4.5$} & \multicolumn{2}{|c|}{$1: 4.6$} \\
\hline Recordings $N\left[\cdot 10^{3}\right]$ & 12 & 24 & 44 & 21 & 21 \\
\hline
\end{tabular}

Figure 5 shows an example of the variation of temperature (orange) and static pressure (black) measured at the base during a wind tunnel run, the shut-down process and thereafter. The random error of the temperature determination was $\Delta T=0.05 \mathrm{~K}$.

The following procedure ensured a synchronized imageand sensor-data acquisition: After the control process for adjusting the flow conditions in the wind tunnel was finished, a trigger signal was sent to start the image capturing, open the lamp shutter and begin the pressure- and temperature-data acquisition. The sensor signals were acquired using a DEWE-50-PCI parallel scanning device. All individual components already worked in loop- or c-W mode to ensure balanced operating conditions.

The intensity images and sensor data were extracted from the continuous time-series, as schematically shown in Fig. 5 during the flow state (wind-on images) and at the reference state (wind-off images) after the shut-down. Dark

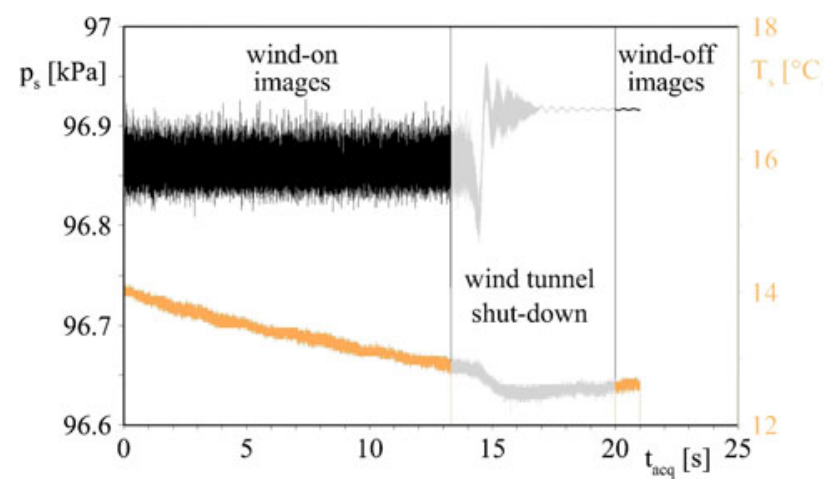

Fig. 5 Schematic to clarify the typical behavior of static base pressure (black) and temperature (orange) during a wind tunnel run. The image acquisition sequence for capturing wind-on/wind-off images is included images, which were used for dark-frame correction of both, the wind-on and the wind-off images, were taken afterward. The PSP sample rates were adjusted according to the freestream velocity in order to resolve an expected reduced frequency of $f \cdot D / U_{\infty}=\mathrm{St}_{\mathrm{D}}=0.21$. They were chosen as $f_{\mathrm{s}, \mathrm{iPSP}}=[1,2,4] \mathrm{kHz}$. At higher frame rates, it became necessary to crop the frame size in order to increase the sampling rate of the camera while performing the image acquisition according to the outlined procedure. More details like frame sizes, fields of view or magnifications can also be found in Table 1 .

\subsubsection{Data evaluation}

Data analysis was performed with the tool IRES -Intensity Reduction \& Evaluation Software developed at the Institute of Fluid Mechanics and Aerodynamics using the Matlab programming language. The structure of this tool offers the possibility to project the entire signal image series automatically on a grid for post-processing and/ or visualization purposes. Several algorithms for image alignment and registration are proposed, see Bell and McLachlan (1996), Sant et al. (1997), to map the intensity images onto three-dimensional grids by using well-known marker distributions and the correspondences in the image plane. These algorithms introduce an artificial noise to the final result, basically originating from sub-pixel misalignment due to model motion between flow and reference condition. Figure 6 shows an image registration analysis of the introduced noise in the intensity ratio $I / I_{0}$ for different artificial model motion amplitudes between 1 and $10 \mathrm{px}$. The wind-on images were sinusoidally shifted around the image center in the $x$ - and $y$-directions with the corresponding amplitudes. The motion-sine-wave was superimposed with $3 \%$ gaussian noise. The $2 \mathrm{k}-\mathrm{by}-2 \mathrm{k} \mathrm{px}^{2}$ 


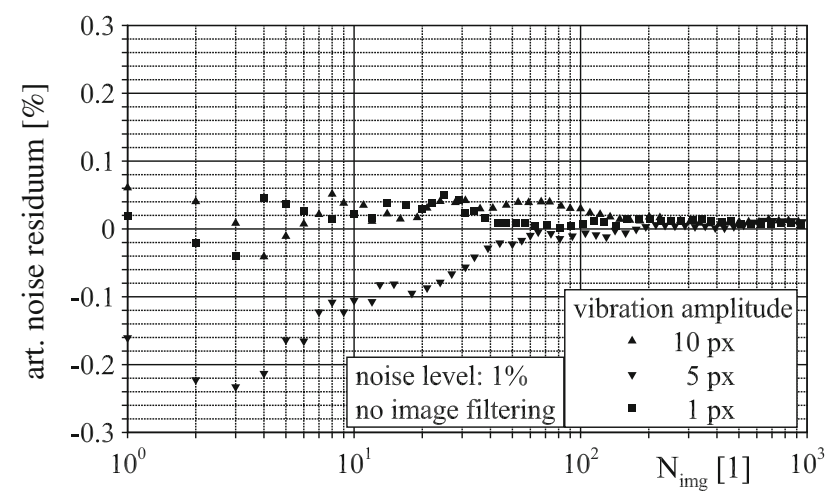

Fig. 6 Residuum of the artificial noise introduced in the intensity ratio due to synthetic model vibration from 1 to $10 \mathrm{px}$ for an IRES evaluation of $N=1,000$ images, global image noise: $1 \%$, no image filtering applied

synthetic intensity images consisted of a constant flat-field value to which a noise level of $1 \%$ was added. The windon/ wind-off ratio of the flat-field parts was 1 . The evaluation was performed with IRES while the residuum of the artificial noise in the intensity ratio $\left(1 / N \cdot \Sigma\left(I_{\mathrm{N}} / I_{0}-1\right)\right.$. $100 \%)$ at the image center was plotted for a set of $N=1,000$ images. The noise is $\leq 0.06 \%$ for a single image and a model motion amplitude $\leq 5 \mathrm{px}$. With a PSP pressure sensitivity of $54 \% / 100 \mathrm{kPa}$, this deviation would result in an artificial pressure error of about $100 \mathrm{~Pa}$. For an intensity ratio averaged over 200 samples, the error is reduced below $20 \mathrm{~Pa}$.

During the data evaluation of the base-measurements (test series 2), the in-situ pressure correction is applied automatically. Therefore, the intensity data are extracted in the vicinity of the pressure transducers, converted into pressure and plotted versus the actual surface pressure given by the transducers. The resulting fit function was applied to the entire intensity image. An average in-situ correction from the base measurement was applied to the wake-intensity images due to the absence of pressure transducers in the sting mount. The typical data evaluation of $N=16,000$ PSP signal images took less than $2 \mathrm{~h}$ with 12 parallel CPUs.

\section{Results}

\subsection{Setup vibration treatment}

A stable illumination pattern is essential for the precise determination of surface pressure distributions with a single component PSP, granted that a stable surface temperature is achieved. The influence of characteristic dynamics of the infrastructure (e.g. vibrations of the wind tunnel and the light source, motion model, etc.) was analyzed in order to separate their impacts on the fluid-dynamic characteristics.
The rear-sting mounting of the model is not a rigid suspension. For a qualification of the models pitching behavior in the test section, the longitudinal and lateral motion was analyzed in the $x$ - $y$ - and in the $y$-z-plane by tracking a model marker, which is used for image resection. The results are presented in Fig. 7. Figure 7a shows the vibration amplitude of the tracked marker in all directions for both Mach numbers. Figure $7 \mathrm{~b}$ shows the power-spectral density of the model motion in the corresponding axes. It could be shown that the model performed a spiral motion in the $y$-z-plane with a maximum amplitude of $\pm 0.5 \mathrm{~mm}$ or $\pm 5.5 \mathrm{px}$. A peak at $f_{\mathrm{r}}=39.2 \mathrm{~Hz}$ characterizes the resonance frequency of the model. Due to this spiral motion, an indirect shifting of the illumination pattern appeared between the wind-on and wind-off states. It was also shown that the amplitudes of the lateral motion in the $z$-direction (green spectrum) are approximately two orders of magnitude higher compared to the motion in the $x$ - and $y$-directions. In this case, the tracking data were extracted from a measurement sequence performed with the second setup. Obviously, the spectrum of the model is

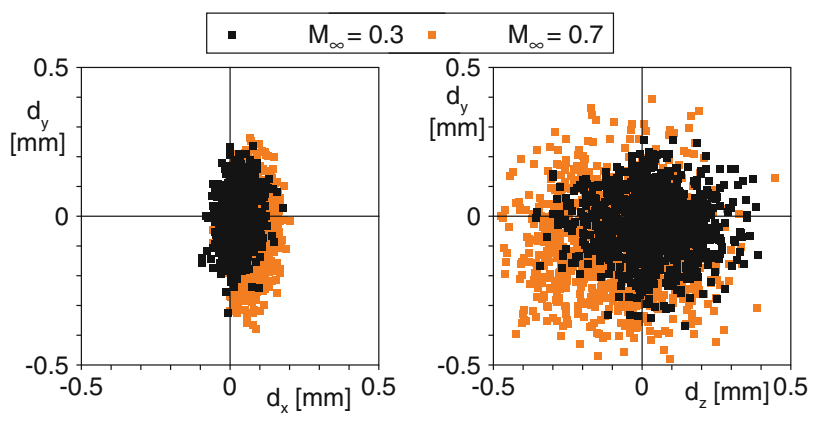

(a)

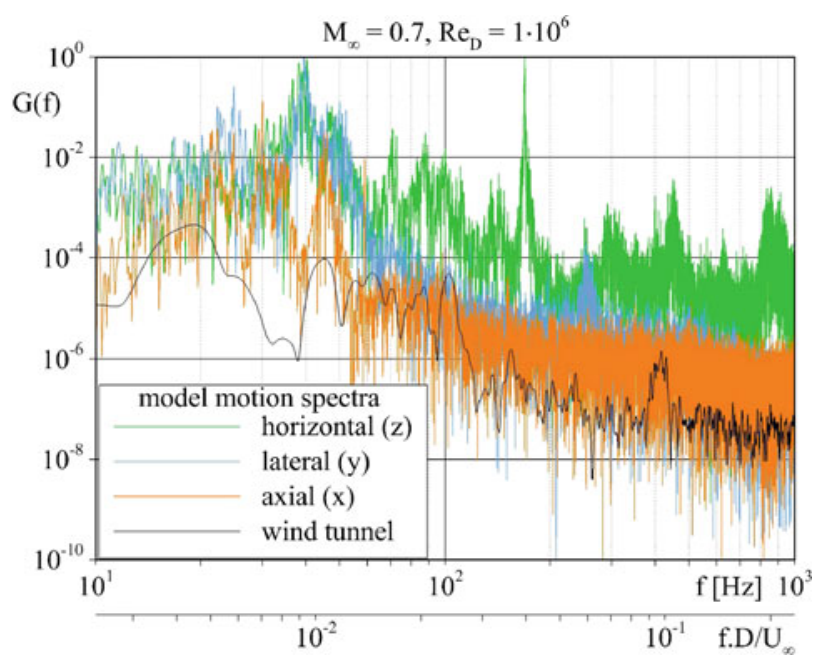

(b)

Fig. 7 Model motion amplitude (a) and power spectra (b) during a wind tunnel run extracted from a tracked marker on the surface of the model. The resonance frequency of the model is $f_{\mathrm{r}}=39.2 \mathrm{~Hz}$ 
superimposed by the vibrating wind tunnel. The camera and the mirror were attached to the wind tunnel walls in this setup, compare Fig. 4. This leads to dominant peaks around the model's resonance frequency and around $f \approx 170 \mathrm{~Hz}$.

The wind tunnel wall was tracked without the model being mounted in the test section for Mach numbers $0.3 \leq \mathrm{M}_{\infty} \leq 2.6$ for an isolated assessment of the wind tunnel vibration. The wind tunnel spectrum at $\mathrm{M}_{\infty}=0.7$ is represented by the black curve in Fig. 7. The wind tunnel generally vibrates at low frequencies around $10-50 \mathrm{~Hz}$ but also has a significant peak around $100 \mathrm{~Hz}$, including its higher harmonics around 200 and $400 \mathrm{~Hz}$. The spectrum remains constant at frequencies $f>100 \mathrm{~Hz}$ for different Mach numbers. Unfortunately, the peak around $f \approx 400 \mathrm{~Hz}$ goes together with the frequency of dominant vortex shedding at $\mathbf{M}_{\infty}=0.3$. It is expected that this perturbation is somehow connected with the hydraulic valve system of the wind tunnel, which controls the total pressure. The pressure perturbation caused by this mechanical device, operating at a fixed frequency, is superimposed to all frequencies measured within the wind tunnel facility.

The illumination pattern, generated by the LED, was synthetically studied in order to quantitatively estimate the effect of model motion on the error in the intensity ratio. Therefore, the camera and an LED with a spherical lens were positioned in front of a diffuse white screen. The working distance and the frame size were chosen according to setup 2 (compare Fig. 4). The intensity map generated by the LED is shown on the left side of Fig. 8. The LED with the lens in front produces a homogeneous but not perfect symmetric illumination pattern. Toward the edge, an intensity increase occurs due to optical distortions caused by lens surface inhomogeneity. The intensity pattern of the LED was synthetically shifted horizontally and
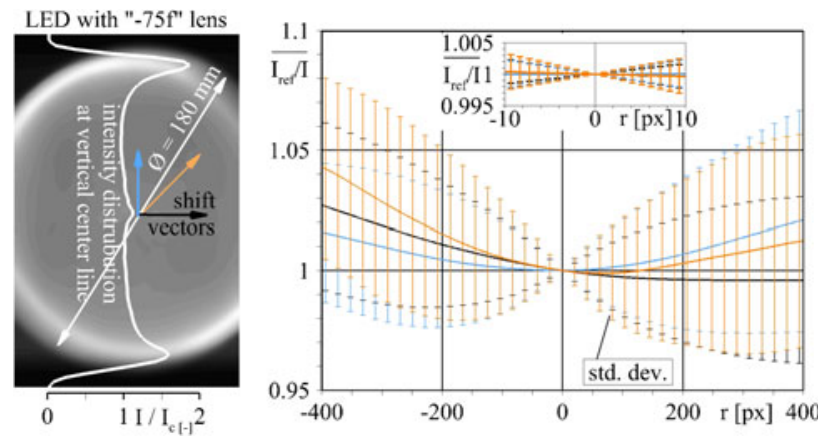

Fig. 8 Synthetic analysis of the error in the Stern-Volmer ratio caused by illumination change due to model motion. The LED illumination pattern (left) was shifted: horizontally (black), vertically (blue), combined (orange). The average intensity ratio $\overline{I_{\text {ref }} / I}$ was evaluated within 11-by-11 px in the image center. The error bars represent the standard deviation vertically within a range of $-200 \leq \mathbf{r} \leq 200$ px with $\Delta \mathbf{r}=$ $0.2 \mathrm{px}$ as the step size. The intensity ratio between the shifted and the non-shifted reference pattern was calculated in the center of the reference pattern within a box of 11-by11 px. The results are also shown in Fig. 8 for the horizontal (black), the vertical (blue) and the combined shifting (orange). The solid lines show the average intensity ratio $\overline{I_{\text {ref }} / I}$ and the error bars represent the corresponding standard deviation within the evaluation region. Their slopes are asymmetric due to the non-rotationally symmetric intensity pattern and because of a slight misalignment of the optical axis between the LED and the lens.

The error in the intensity ratio caused by the actual model motion of $\pm 5.5 \mathrm{px}$ during the experiments can be assessed from the detailed view in Fig. 8. It introduces an additional measurement uncertainty in the intensity ratio of $0.023 \pm 0.17 \%$ or approximately $350 \mathrm{~Pa}$.

\subsection{Comparison: iPSP versus pressure transducers}

At first, a comparison between the signals from the conventional unsteady pressure transducers and the results of the iPSP measurements is made, in order to ensure that characteristic frequencies, which were resolved with iPSP, reliably represent the flow topology. An artificial binning, comparable to the PSP integration time, of the $10 \mathrm{kHz}-$ pressure transducer signal was made in order to compare the power spectrum (PSD-G(f)) of the PSP signals to the ones from the transducers with the same resolution in time. Figure 9 shows the resulting PSD of an original pressure transducer signal (blue) and of the same signal adapted to an iPSP sampling rate of $2 \mathrm{kHz}$ (black). The noise from the original signal is slightly reduced, whereas all features are kept in the band that iPSP resolves.

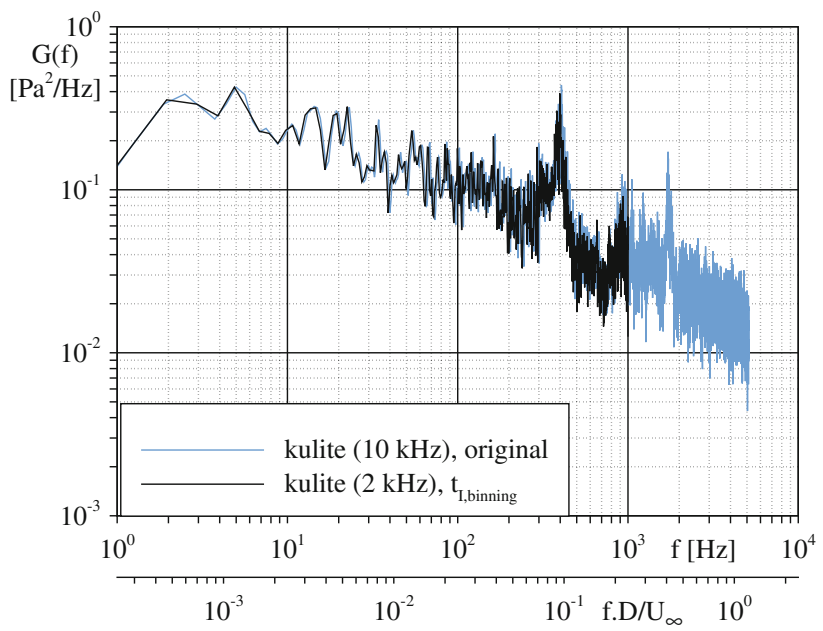

Fig. 9 Comparison of the power-spectral density $G(f)$ for an original pressure transducer signal (blue) and for an iPSP-adapted transducer signal (black) 


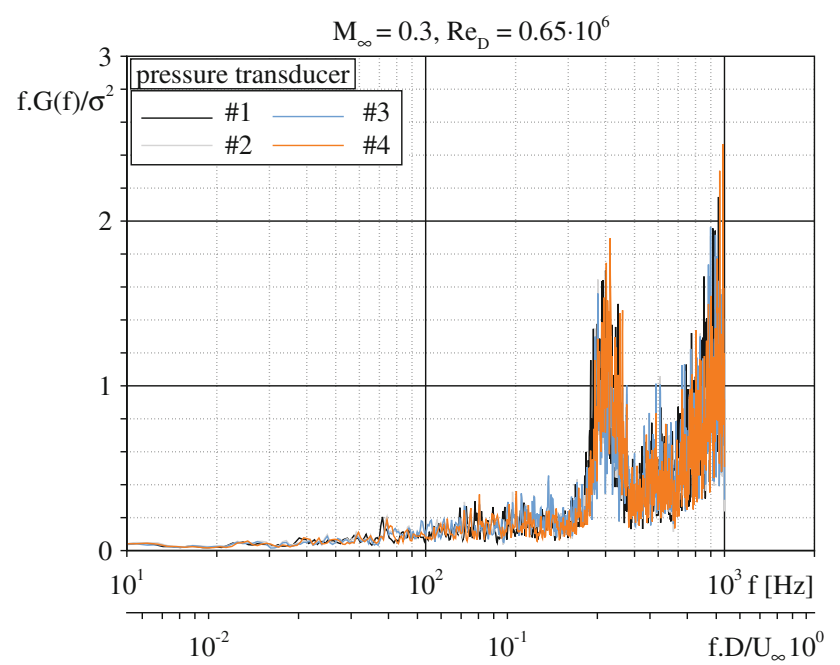

Fig. 10 Comparison of the premultiplied power-spectral density $f \cdot G(f) / \sigma^{2}$ of the 4 active pressure transducers in the base of the model located at $r / R=[0.45 ; 0.52 ; 0.85 ; 0.95]$

Figure 10 shows the similarity of the 4 available pressure transducers. Only the 3rd sensor located at $r / R=0.52$ is used as reference in the following discussions due to this similarity.

$\sigma^{2}=\int_{0}^{\infty} f \cdot G(f) d[\log (f)]=c_{\mathrm{p}, \mathrm{rms}}^{2}$

The power spectrum $G(f)$, which is normalized by $f / \sigma^{2}$ (Owen (1958)), represents the total amount of energy caused by the pressure fluctuation in the corresponding frequency domain. The integrated values for $c_{\mathrm{p}, \mathrm{rms}}$ are

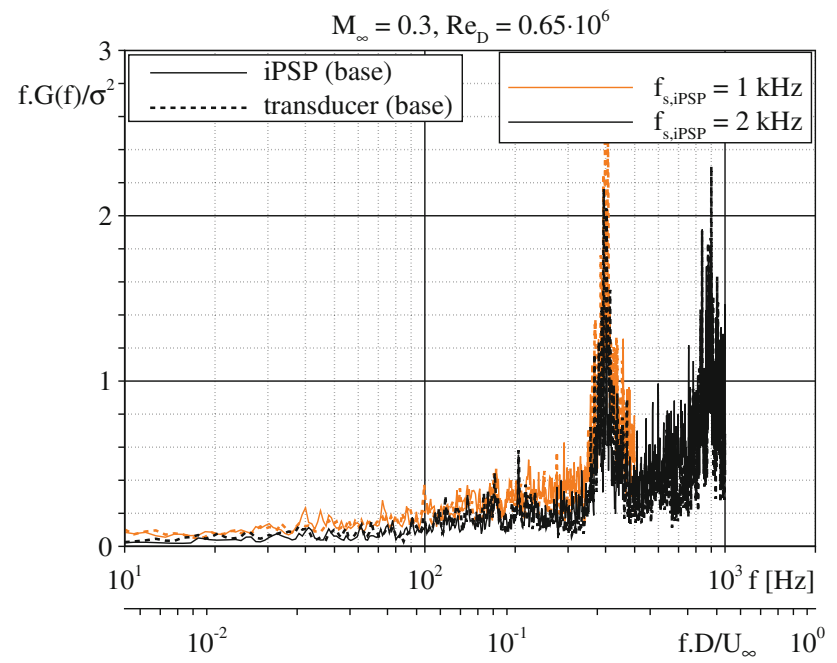

Fig. 11 Comparison of base buffet spectra at $\mathbf{M}_{\infty}=0.3$ derived from the iPSP signal (solid) and from the unsteady pressure transducers (dashed). The iPSP sampling rates were $f_{\mathrm{s}, \mathrm{PSP}}=1 \mathrm{kHz}$ (black) and $f_{\mathrm{s}, \mathrm{iPSP}}=2 \mathrm{kHz}$ (orange) equal to $c_{\mathrm{p}, \mathrm{rms}}=\sqrt{\overline{p^{\prime 2}}} / q_{\infty}$, whereas $p^{\prime}$ is the fluctuation pressure. The power spectrum itself was obtained by applying a windowed Welch algorithm implemented in Matlab. The overlap between the individual Welch-windows was $75 \%$. Each window had a length of $1 \mathrm{~s}$ (e.g. 1,000 or 2,000 samples, according to $\left.f_{\mathrm{s}, \mathrm{PPSP}}\right)$. In Figs. 11 and 12, the comparison between the transducer (dashed) and iPSP (solid) spectra on the base is shown at $\mathrm{M}_{\infty}=0.3$ and $\mathrm{M}_{\infty}=0.7$. The different iPSP sampling rates are color-coded. A good agreement between the spectra in both the amplitude and the position of characteristic frequencies shows that iPSP is capable of resolving the flow phenomena with small amplitudes precisely. In Fig. 11, a dominant peak establishes around $f \approx 405 \mathrm{~Hz}$ and a second, wider one around $f \approx 870 \mathrm{~Hz}$. The first peak confirms the expected frequency of characteristic vortex shedding at a reduced frequency of $f \cdot D / U_{\infty}=0.21$. The second, wider peaks ought to represent its higher harmonic, as they were already measured in Scharnowski and Kähler (2011). In the case of $\mathrm{M}_{\infty}=0.7$, some dominant peaks form around $f \approx 400 \mathrm{~Hz}$ and around $f \approx 905 \mathrm{~Hz}$. The peak around $f \approx 905 \mathrm{~Hz}$ corresponds to the characteristic vortex shedding frequency, while the peak around $f \approx 400 \mathrm{~Hz}$ corresponds to the peak in the spectrum of the wind tunnel caused by the hydraulic system. The final in-situ pressure errors were about 5-7 \% (higher at $\mathrm{M}_{\infty}=0.3$ due to a higher static pressure and a smaller pressure gradient). It was slightly larger than the expected uncertainty from the combination of the PSP-temperature effect, the registration noise of a single image and the model motion of $5.25 \%$. Additional factors might be paint contamination and aging.

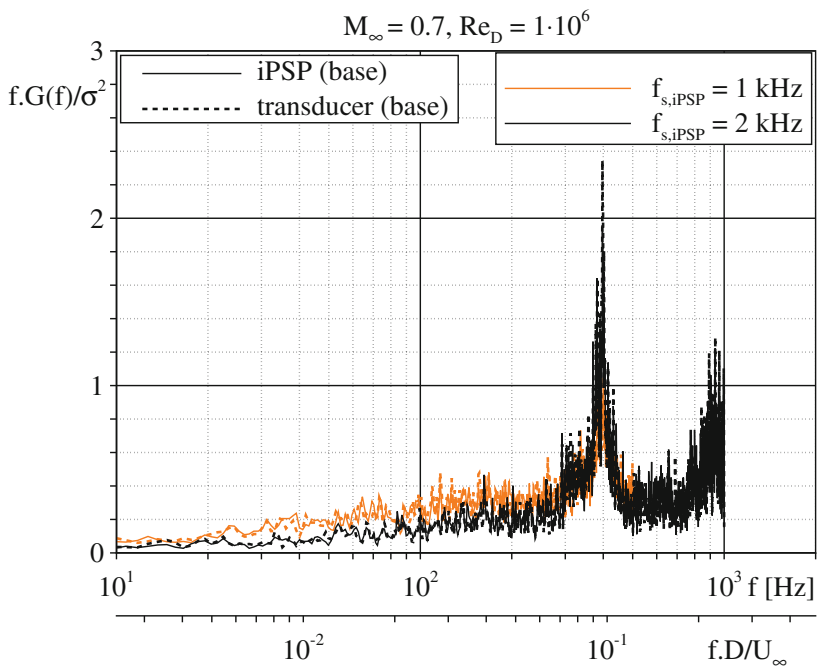

Fig. 12 Comparison of base buffet spectra at $\mathrm{M}_{\infty}=0.7$ derived from the iPSP signal (solid) and from the unsteady pressure transducers (dashed). The iPSP sampling rates were $f_{\mathrm{s}, \mathrm{PSP}}=1 \mathrm{kHz}$ (black) and $f_{\mathrm{s}, \mathrm{PSP}}=2 \mathrm{kHz}$ (orange) 


\subsection{Base flow characterization}

\subsubsection{Pressure distribution}

The ensemble-averaged base pressure distributions are shown in Figs. 13 and 14 for $\mathrm{M}_{\infty}=0.3$ and $\mathrm{M}_{\infty}=0.7$ respectively. The base view from the rear is presented in polar coordinates $(\Phi, r / R)$, whereas $\Phi=0^{\circ}$ represents the positive $y-$ axis in the global coordinate system. The gray-filled areas cover screws, which were needed to attach the base to the rest of the model as well as the pressure transducers, where no iPSP signal is available. The righthand side of each figure shows a line plot of $c_{\mathrm{p}}$ (solid) and $c_{\mathrm{p}, \mathrm{rms}}$ (dashed) extracted at $\Phi=55^{\circ}$ and compares it to the pressure transducer data. The values of $c_{\mathrm{p}, \mathrm{rms}}$ are nearly doubled in case of the subsonic Mach number compared to the transonic one. The reason for this might be the flow quality of the wind tunnel, which operates with a turbulence level of $T u=1.2 \%$ at its lower Mach number limit. The values of $c_{\mathrm{p} \text {,rms }}$ reach their maximum in the area of $c_{\mathrm{p}, \mathrm{min}}$. An obvious pressure change toward the sting $(r / R=0.4)$ is caused by a secondary vortex rotating in the junction between the sting and the model base. Its asymmetric distribution, especially at $\mathbf{M}_{\infty}=0.7$, might indicate a slightly oblique oncoming flow due to sting bending or model installation. Unfortunately, the 4 installation pressure ports on the cylindrical part of the model indicated no difference in the static pressure. This avoided a potential intervention and correction of the angle of attack.

\subsubsection{Pressure fluctuation characteristics}

The auto-correlation analysis from the time signal of the radial pressure fluctuation on the base at $\mathbf{M}_{\infty}=0.3$ was made for the identification of dominant time scales, as suggested by Mabey (1972). Therefore, the pressure fluctuation signal was extracted at $r / R=0.52=$ const. The left-hand side of Fig. 15 shows a contour map of the autocorrelation coefficient $R$. A homogeneously distributed correlation peak is expected for the turbulent separating flow at the base. The actual distribution is homogeneous over a wide radial range. Around $\Phi=0^{\circ}$ the auto-correlation peak is spreading slightly, which indicates a variation in the dominant time scales. This might be caused by wall interferences due to the rectangular cross-section dimensions of the wind tunnel. A cross-correlation between the radial pressure fluctuation signals and the reference signal at $\Phi=0^{\circ}$ is shown on the right-hand side of Fig. 15. This method can be used for an identification of potential flow pattern convection rates, see Hudy et al. (2007). The inclination of the correlation peak is a measure for convection rates of flow patterns. A segmentation of the crosscorrelation plane was made for estimating its orientation using a threshold of $R=0.1$ as indicated by the black border in Fig. 15. The angle of the main peak was estimated by an elliptical fitting. The ellipse's inclination can be calculated from its major and minor axes' orientations. By applying this procedure, a reduced convection rate of $f_{\mathrm{c}}$. $U_{\infty} / D \approx 0.071$ was calculated. Figure 16 shows the spacetime correlation of the same radial pressure fluctuation signal for 200 reduced time steps $\tau \cdot U_{\infty} / D$. This plot allows the identification of convecting pattern with a reduced convection rate of $f_{\mathrm{c}} \cdot U_{\infty} / D \approx 0.079$ (black arrows). This reduced convection rate correlates well with the convection rate estimated by the previous correlationpeak inclination analysis. It suggests a radial convection velocity of $\omega=2 \pi f_{\mathrm{c}} \approx 950 \mathrm{rad} / \mathrm{s}$. As a result of the chosen sampling rates, a convection-rate analysis for $\mathrm{M}_{\infty}=0.7$ provided no satisfying result.

Large-eddy flow simulations, which were performed by project partners (Statnikov et al. (2012)), indicated a modelike $60^{\circ}$-distribution of the base pressure fluctuations $p^{\prime}$. In order to identify a potential dominant radial wavelength, the pressure fluctuation signal shown in Fig. 16 was Fouriertransformed, and its inverse frequency was calculated. The results are presented in Fig. 17. Structures with large wavelength dominate the spectra. The formation of pressure modes with a preferred $60^{\circ}$-distribution could not be confirmed by this analysis. However, a mode-like appearance of

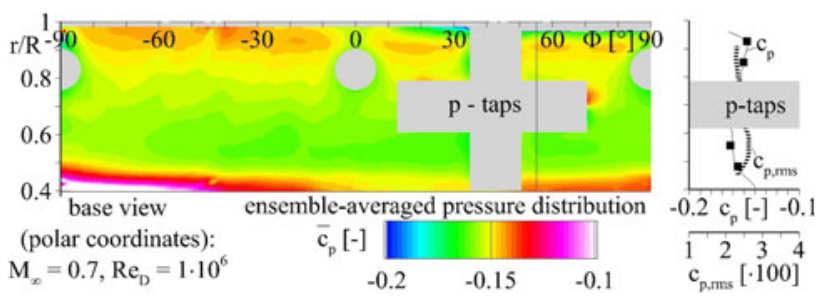

Fig. 14 Left ensemble-averaged base pressure distribution at $\mathrm{M}_{\infty}=$ 0.7 in polar coordinates $(\Phi, r / R)$; right: line plots of $c_{\mathrm{p}}$ and $c_{\mathrm{p}, \mathrm{rms}}$ extracted at $\Phi=55^{\circ}$ in comparison with pressure transducers (square symbols)
Fig. 13 Left: ensemble-averaged base pressure distribution at $\mathbf{M}_{\infty}=$ 0.3 in polar coordinates $(\Phi, r / R)$; right: line plots of $c_{\mathrm{p}}$ and $c_{\mathrm{p}, \mathrm{rms}}$ extracted at $\Phi=55^{\circ}$ in comparison with pressure transducers (square symbols) 

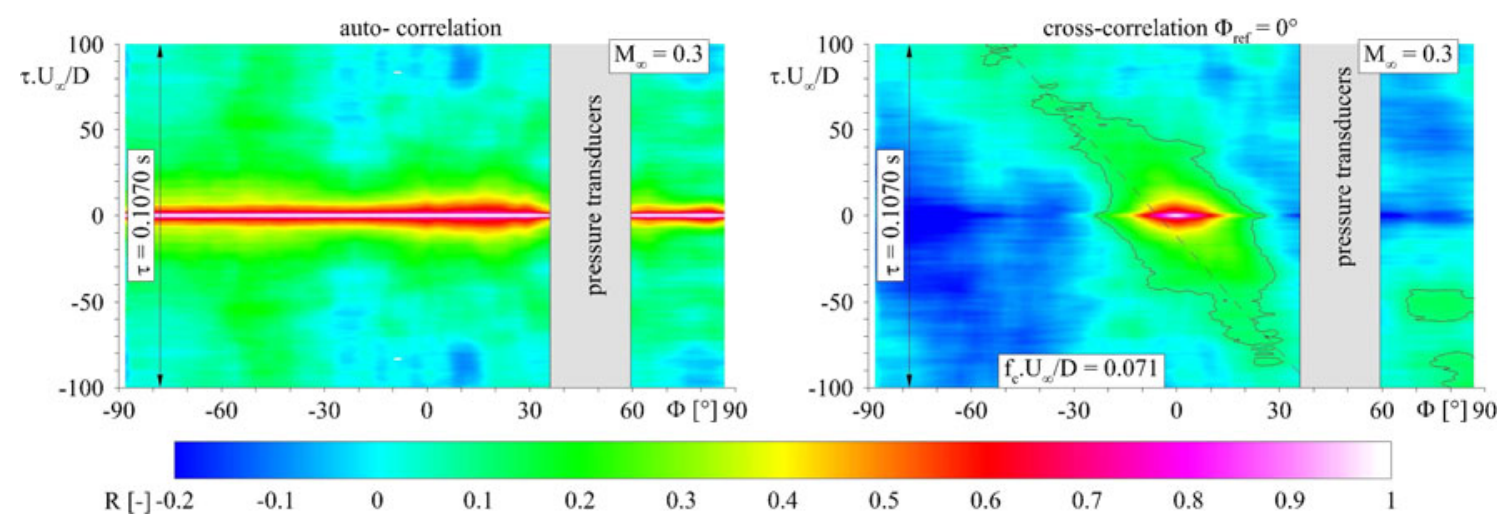

Fig. 15 Left contour map of the auto-correlation coefficient $R$ for all base pressure fluctuation signals along $r / R=0.52$; right: contour map of the cross-correlation coefficient $R$ for all base pressure fluctuation

signals along $r / R=0.52$ with the reference signal at $\Phi=0^{\circ}$. Correlation-peak threshold $=0.1$ (black border); mean pattern convection $f_{\mathrm{c}} \cdot U_{\infty} / D$ (inclined dashed line)

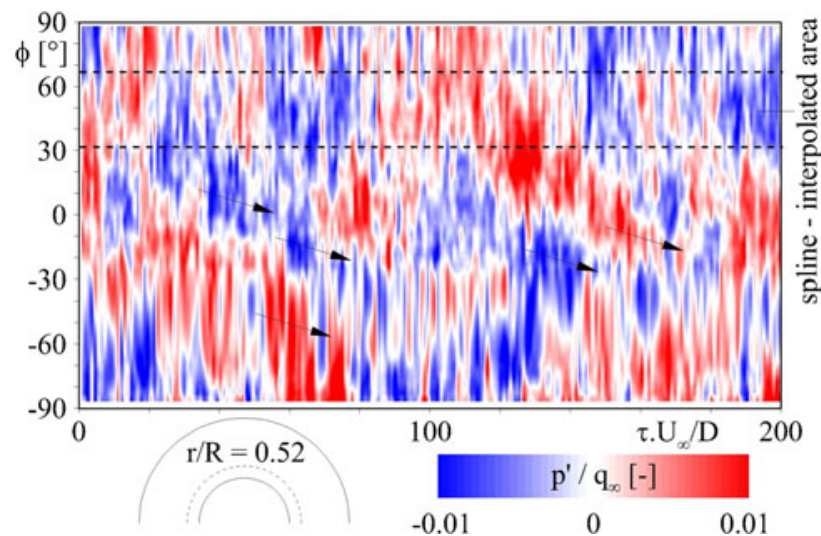

Fig. 16 Space-time correlation plot of the normalized pressure fluctuation $p^{\prime} / q_{\infty}$ at $\mathrm{M}_{\infty}=0.3$ extracted from the base at $r / R=0.52$ for 200 reduced time steps $\tau \cdot U_{\infty} / D$. The black arrows indicate pattern convection with a reduced convection rate of $f_{\mathrm{c}} \cdot U_{\infty} / D=0.079$

$p^{\prime}$ is somehow present. Its wavelengths seemed to be around $100^{\circ} \leq \lambda_{\mathrm{p}^{\prime}} \leq 200^{\circ}$ as indicated by both Figs. 16 and 17.

\subsection{Wake flow characterization}

After demonstrating the potential of optical pressure measurements in comparison with conventional pressure transducers, the iPSP data are now discussed as stand-alone results for an analysis of the surface pressure distributions and the characterization of the wake-buffet spectra.

\subsubsection{Pressure distribution}

Figures 18 and 19 show a contour map of the ensembleaveraged wake pressure distribution at $\mathrm{M}_{\infty}=0.3$ and $\mathrm{M}_{\infty}=0.7$ acquired at $2 \mathrm{kHz}$ and averaged from 16384 wind-on samples. The line plots of $c_{\mathrm{p}}$ and $c_{\mathrm{p}, \mathrm{rms}}$ in the upper part were extracted at $\Phi=-200^{\circ}$ as indicated by the black

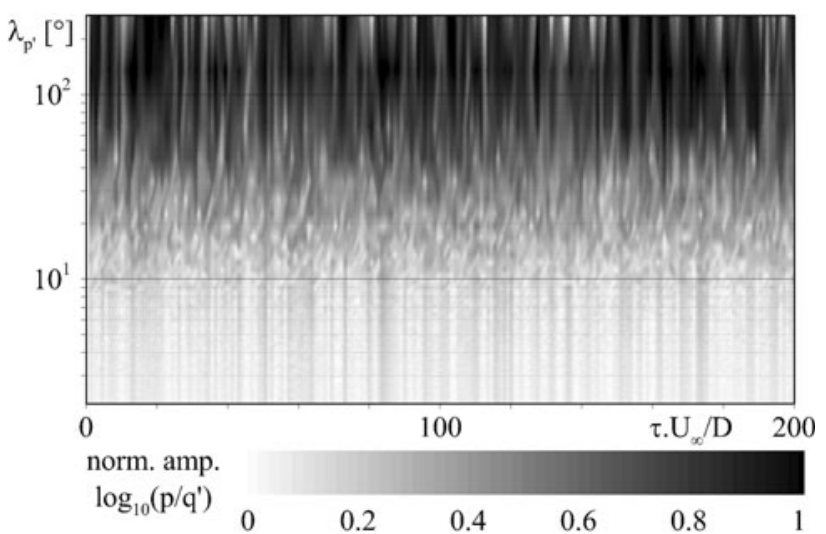

Fig. 17 Spatial wavelengths $\lambda_{p^{\prime}} \propto 1 / f$ of the pressure fluctuation on the base extracted at $r / R=0.52$ for 200 reduced time steps $\tau \cdot U_{\infty} / D$

trace in the surface pressure plot. The axial coordinate was normalized with the corresponding reattachment lengths, which were $l_{\mathrm{r}}=[1.17 ; 1.33] x / D$ at $\mathrm{M}_{\infty}=[0.3 ; 0.7]$. At $\mathrm{M}_{\infty}=0.3$, the difference in $c_{\mathrm{p}}$ between the minimum and the maximum is quite high. This might indicate that the pressure perturbation caused by the wind tunnel somehow dominates the wake flow at this Mach number. A second indicator for this might be the values of $c_{\mathrm{p}, \mathrm{rms}}$, which are nearly doubled, compared to the ones from $\mathrm{M}_{\infty}=0.7$. The formation of locally predefined low pressure regions in the ensemble-averaged pressure distribution closely behind the base also confirms the assumption that the wake flow is somehow triggered and not fully developed at $\mathrm{M}_{\infty}=0.3$. The dot-like structures, which appear in the contour map, especially in Fig. 18, originate from the image resection markers. In the case of $\mathrm{M}_{\infty}=0.7$, the line plots of $c_{\mathrm{p}}$ and $c_{\mathrm{p}, \mathrm{rms}}$ are compared to data from Deprés et al. (2004), whose data were acquired with unsteady pressure transducers at $\mathrm{M}_{\infty}=0.85$ on a comparable geometry, equipped with a cylindrical nozzle of length $1.2 \mathrm{D}$ instead of a rearsting mounting. The good agreement in both $c_{\mathrm{p}}$ and $c_{\mathrm{p}, \mathrm{rms}}$ 


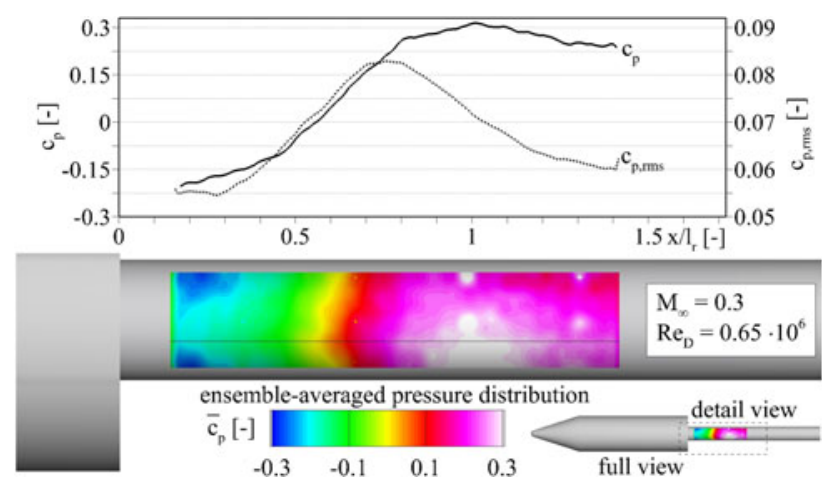

Fig. 18 Bottom Ensemble-averaged stream-wise wake pressure distribution $\bar{c}_{\mathrm{p}}$ at $\mathrm{M}_{\infty}=0.3$; top line plots of $c_{\mathrm{p}}$ and $c_{\mathrm{p}, \text { rms }}$ extracted at $\Phi=-200^{\circ}$

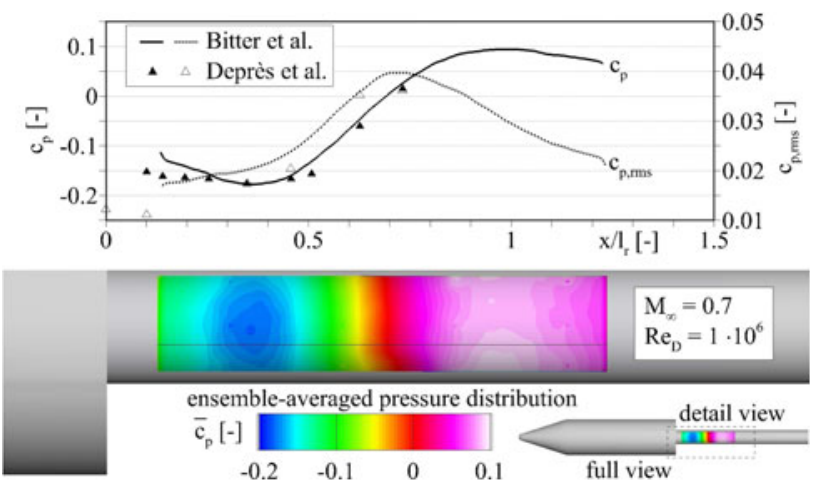

Fig. 19 Bottom ensemble-averaged stream-wise wake pressure distribution $\bar{c}_{\mathrm{p}}$ at $\mathrm{M}_{\infty}=0.7$; top line plots of $c_{\mathrm{p}}$ and $c_{\mathrm{p}, \text { rms }}$ extracted at $\Phi=-200^{\circ}$ and compared with data from Deprés et al. (2004) included (triangles)

validates the fact that a high-resolution estimation of pressure fluctuations with small amplitudes is possible by using fast-responding pressure-sensitive paints. It is confirmed, as shown in experiments by Hudy et al. (2007) or numerical simulation by Deck and Thorigny (2007), that the location of highest $c_{\mathrm{p}, \mathrm{rms}}$ is located slightly upstream of the mean reattachment length $l_{\mathrm{r}}$.

\subsubsection{Buffeting}

For an estimation of the strength and potential dynamics of coherent wake pressure fluctuations, the contour maps of the stream-wise buffet spectra are shown in Figs. 20 and 21 for $\mathrm{M}_{\infty}=0.3$ and $\mathrm{M}_{\infty}=0.7$, respectively. The evaluation was done similarly to the one presented in Sect. 3.3 using the Welch algorithm at each axial position of the mapped pressure data extracted at $\Phi=-200^{\circ}$. It is evident from both figures that the characteristic frequencies of dominant vortex shedding spread out along the entire wake nearly independent of the axial position. The broad-band spectra

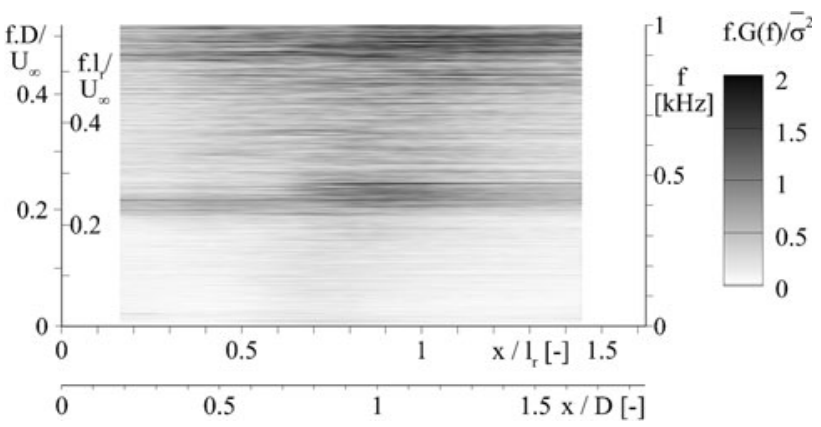

Fig. 20 Contour map of the wake-buffet spectrum at $\mathbf{M}_{\infty}=0.3$. Axial coordinate $x$ normalized with the model diameter $D$ and the reattachment length $l_{\mathrm{r}}$. Dominant vortex shedding occurs around $f_{\text {shed }} \approx 0.40 \mathrm{kHz}$

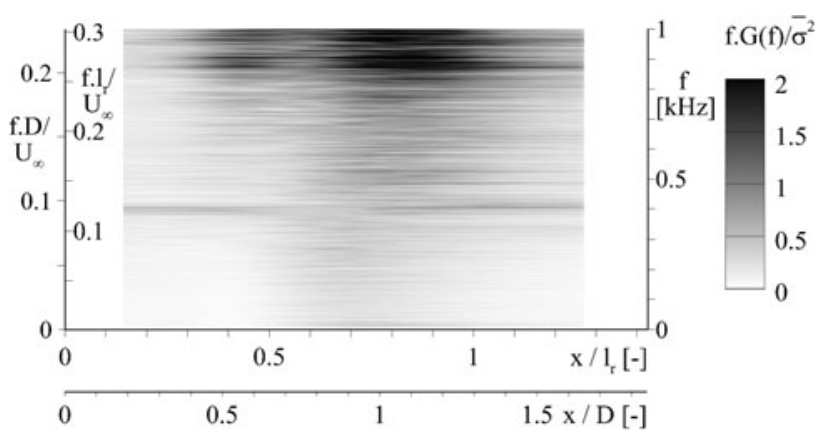

Fig. 21 Contour map of the wake-buffet spectrum at $\mathbf{M}_{\infty}=0.7$. Axial coordinate $x$ normalized with the model diameter $D$ and the reattachment length $l_{\mathrm{r}}$. Dominant vortex shedding occurs around $f_{\text {shed }} \approx 0.90 \mathrm{kHz}$

contain no distinct peaks at the frequencies of vortex shedding, but a clear indication of events occurring around a reduced frequency of $f \cdot U_{\infty} / D \approx 0.21$. Apparently, the spectrum is somehow intermittent in the case of $\mathrm{M}_{\infty}=0.7$. The region of high dynamic range in the core region of the mean wake vortex is obviously produced by smaller eddys, since large-scale structures should be too inert to follow the flow in this area. A second domain around $x / D \approx 1$ originates from the large-scale shear layer structures that clash on the sting and decay into smaller structures. These structures dominate the wake flow as already indicated by the slopes of $c_{\mathrm{p}, \mathrm{rms}}$ in the previous section. As also visible from the figures, the perturbation around $f \approx 400 \mathrm{~Hz}$, caused by the wind tunnel, is present along the entire nearwake.

\subsection{Comparison: experiment versus CFD}

Finally, a comparison of the flow topology in the nearwake of the generic spacecraft model, determined by modern experimental (Fig. 22) and numerical methods 


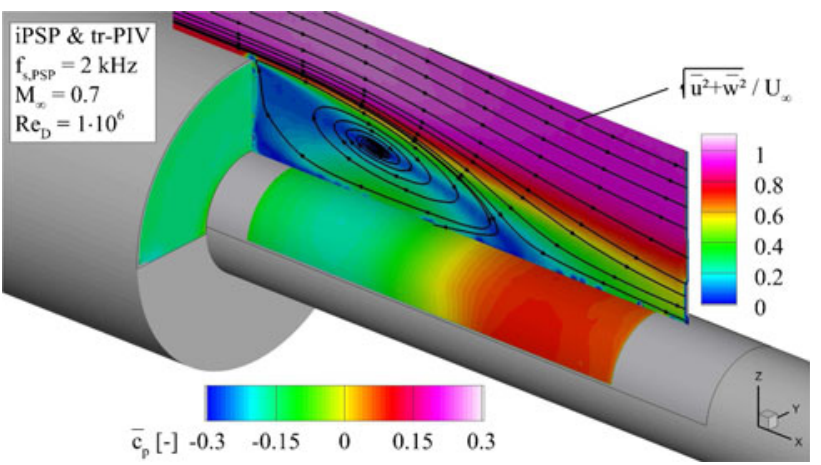

Fig. 22 Flow field in the near-wake region gathered with experimental methods. The pressure distribution on the sting and the base was measured with fast-responding pressure-sensitive paint and the velocity magnitude in the $x-z-$ plane was acquired with timeresolved particle-image velocimetry (data from Bitter et al. (2011))

(Fig. 23), is presented. On the surface of the model, the color-coded ensemble-averaged static pressure distributions $c_{\mathrm{p}}$ are displayed. Additionally, the normalized, absolute flow velocities are presented color-coded in the $x$-z-plane at $y=0$ (the iPSP results were rotated for display purposes in this figure). The experimental data came from the experiments presented in this work and from time-resolved particle-image velocimetry measurements (tr-PIV), see Bitter et al. (2011). The results of both test series correlate very well with each other, whereas the position of the mean reattachment differs between $l_{\mathrm{r}}=1.33 x / D$ (iPSP) and $l_{\mathrm{r}}=1.25 x / D$ (tr-PIV). This indicated a slight difference in the Reynolds number. The streamlines precisely reflect both the position of the center of the averaged wake vortex, as well as the reattachment position on the nozzle (the rear-sting mount).

The numerical results in Fig. 23 reflect the ensembleaveraged data, which was extracted from large-eddy-simulations (LES) provided by project partners, see Statnikov et al. (2012). The time-averaging was performed over 136 LES time steps. The step size between the individual time steps was $\Delta t=\tau_{\text {ref }} / 10=0.0237 \mathrm{~ms}$. The computation domain was cropped down to a section of $60^{\circ}$, and the grid was split up into several zones (black zone edges) in order to reduce the computation time. The simulations were performed with periodic boundary conditions. A zonal approach with overlapping RANS- and LES-domains was used. The introduction of turbulence started, where the RANS- and the LES-domains overlapped. This position on the cylindrical part of the main body is indicated by the origin of the stream lines in Fig. 23. The node number for the entire mesh was $60 \times 10^{6}$. Further details of the numerical approaches and models can be found in Statnikov et al. (2012). The comparison of both figures shows a good agreement in the magnitudes of the predicted pressure- and velocity fields. The reattachment length is overpredicted by about $25 \%$ by the numerical simulations. This

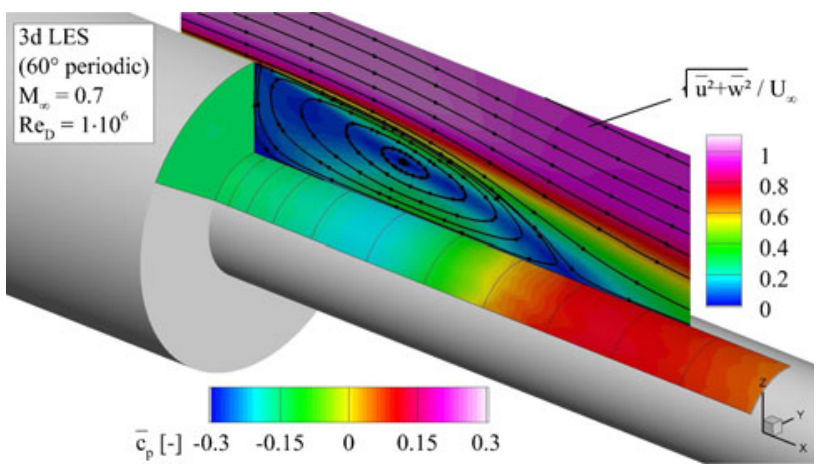

Fig. 23 Flow field in the near-wake region gathered from large-eddysimulation, provided with courtesy of Statnikov et al. (2012)

discrepancy is mainly influenced by the mismatch of the turbulence level $\mu_{\mathrm{t}}$ between the experiment and the simulation. However, for future simulations, the data set presented within this paper can be used for validation purposes.

\section{Conclusions and outlook}

The capabilities of the instationary pressure-sensitive paint (iPSP) technique were presented in order to characterize the separating/reattaching flow around a generic spacecraft model in the Trisonic Wind tunnel Munich (TWM) at freestream Mach numbers $\mathbf{M}_{\infty}=[0.3 ; 0.7]$. Characteristic flow frequencies up to $f=1,000 \mathrm{~Hz}$ were successfully confirmed with high spatial resolution by using a polymerceramic base layer (pc-PSP) in combination with platinum complexes (PtTFPP) as pressure-sensitive dye. The comparison with classical pressure transducers indicated that the measurement uncertainty of $5-7 \%$ is mainly caused by the temperature effect of pc-PSP. With respect to the outlined scientific key questions, the expected characteristic frequencies of dominant vortex shedding were confirmed to be around $f_{\text {shed }} \approx[405 ; 905] \mathrm{Hz}$ at $\mathbf{M}_{\infty}=[0.3 ; 0.7]$. It was also possible to characterize the buffet dynamics in the re-circulation zone, particularly at the reattachment position.

A spectral analysis of the spatial distribution of the flow structures at the base indicates the presence of mode-like pressure fluctuations. However, no evidence for the occurrence of pressure modes with a preferred circumferential $60^{\circ}$-distribution could be detected. Trends indicate the existence of long-wavelength pressure modes of $\lambda_{p^{\prime}} \geq 100^{\circ}$.

The comparison of experimental data with the results from large-eddy-simulations indicates a good agreement between the flow topologies in the near-wake. The spatial extent of the re-circulation domain in the axial flow direction has been determined to be of similar size by independent measurement campaigns using different 
optical measurement techniques. Certainly, the reattachment length is over-predicted by about $25 \%$ by the largeeddy-simulations as a result of the different turbulence levels between experiment and simulations. The established database can be used for the validation of numerical flow simulations in the future.

Acknowledgments Financial support has been partially provided by the German Research Foundation (Deutsche Forschungsgemeinschaft-DFG).

Open Access This article is distributed under the terms of the Creative Commons Attribution License which permits any use, distribution, and reproduction in any medium, provided the original author(s) and the source are credited.

\section{References}

Airaghi SA (2006) Self-illuminating pressure sensitive paints using electroluminescent foils. PhD thesis, ETH Zürich

Asai K, Iijima Y, Kanda H, Kunimatsu T (1998) Novel pressuresensitive coating based on anodic porous alumina. Okayama, Japan, 30th fluid dynamics conference

Asai K, Nakakita K, Kameda M, Teduka K (2001) Recent topics in fast-responding pressure-sensitive paint technology at national aerospace laboratory. In: 19th International congress on instrumentation in aerospace simulation facilities, ICIASF 2001, Cleveland, Ohio, pp 25-36

Bell JH, McLachlan BG (1996) Image registration for pressuresensitive paint applications. Exp Fluids 22:78-86

Bitter M, Klein C, Kähler CJ (2009) PSP und $\mu$ PSP Untersuchungen zur Freistrahl / Wand-Interaktion im Unter- und überschall. In: 17. Fachtagung Lasermethoden in der Strömungsmesstechnik, Deutsche Gesellschaft für Laser-Anemometrie, Erlangen, Germany

Bitter M, Scharnowski S, Hain R, Kähler CJ (2011) High-repetitionrate piv investigations on a generic rocket model in sub- and supersonic flows. Exp Fluids 50:1019-1030

Crafton J, Lachendro N, Guille M, Sullivan JP (1999) Application of temperature and pressure sensitive paint to an obliquely impinging jet. AIAA 1999-0387

Deck S, Thorigny P (2007) Unsteadiness of an axisymmetric separating-reattaching flow: Numerical investigation. Phys Fluids 19(6):065,103

Deprés D, Reijasse P, Dussauge J (2004) Analysis of unsteadiness in afterbody transonic flows. AIAA J 42:2541-2550

Eaton JK (1980) Turbulent flow reattachment: an experimental study of the flow and structure behind a backward-facing step. $\mathrm{PhD}$ thesis, Stanford University

Gregory JW, Sullivan JP, Raman G, Raghu S (2007) Characterization of the microfluidic oscillator. AIAA J 45(3):568-576

Gregory JW, Asai K, Kameda M, Liu T, Sullivan JP (2008) A review of pressure-sensitive paint for high-speed and unsteady aerodynamics. Proc Inst Mech Eng Part G J Aeros Eng 222(2):249-290

Gülhan, A (eds) (2008) RESPACE-Key Technologies for reusable space systems, notes on numerical fluid mechanics and multidisciplinary design, vol 98, Springer, Berlin

Hudy LM, Naguib A, Humphreys W (2007) Stochastic estimation of a separated-flow field using wall-pressure-array measurements. Phys Fluids 19(2):024,103

Juliano TJ, Disotell KJ, Gregory JW, Crafton J, Fonov S (2012) Motion-deblurred, fast-response pressure-sensitive paint on a rotor in forward flight. Meas Sci Technol 23(4):045,303
Kameda M, Tabei T, Nakakita K, Sakaue H, Asai K (2005) Image measurements of unsteady pressure fluctuation by a pressuresensitive coating on porous anodized aluminium. Meas Sci Technol 16(12):2517

Klein C, Sachs WE, Henne U, Egami Y, Mai H, Ondrus V, Beifuss U (2010) Application of pressure-sensitive paint for determination of dynamic surface pressures on a $30 \mathrm{hz}$ oscillating $2 \mathrm{~d}$ profile in transonic flow. In: New results in numerical and experimental fluid mechanics VII, notes on numerical fluid mechanics and multidisciplinary design, vol 112, Springer, Berlin, pp 323-330

Le Sant Y (2001) Overview of the self-illumination effect applied to pressure sensitive paint applications. International congress on instrumentation in aerospace simulation facilities, international congress on instrumentation in aerospace simulation facilities, pp 159-169

Lee I, Sung HJ (2001) Characteristics of wall pressure fluctuations in separated flows over a backward-facing step: Part i. time-mean statistics and cross-spectral analyses. Exp Fluids 30:262-272

Lee I, Sung HJ (2002) Multiple-arrayed pressure measurement for investigation of the unsteady flow structure of a reattaching shear layer. J Fluid Mechan 463:377-402

Liu T, Sullivan JP (2005) Pressure and temperature sensitive paints. Experimental fluid mechanics. Springer, Berlin

Mabey D (1972) Analysis and correlation of data on pressure fluctuations in separated flow. AIAA J Aircraft 9:642-645

Méerienne MC, Sant YL, Ancelle J, Soulevant D (2004) Unsteady pressure measurement instrumentation using anodized-aluminium psp applied in a transonic wind tunnel. Meas Sci Technol 15(12):2349

Nakakita K (2007) Unsteady pressure distribution measurement around 2d-cylinders using pressure-sensitive paint. In: 25th AIAA Applied Aerodynamics Conference, Miami

Owen T (1958) Techniques of pressure-fluctuation measurements employed in the rae low-speed wind-tunnels. AGARD 172

Ruyten WM (1997) Self-illumination calibration technique for luminescent paint measurements. Rev Sci Instrum 68(9):3452-3457

Sakaue H, Tabei T, Kameda M (2006) Hydrophobic monolayer coating on anodized aluminum pressure-sensitive paint. Sens Actuators B Chem 119(2):504-511

Sant YL, Deleglise B, Mebarki Y (1997) An automatic image alignment method applied to pressure sensitive paint measurements. In: International congress on instrumentation in aerospace simulation facilities, pp 57-65

Scharnowski S, Kähler CJ (2011) Investigation of the wake dynamics of a generic space launcher at $\mathrm{Ma}=0.7$ by using high-repetition rate PIV. In: 4th European conference for aerospace sciences, Saint Petersburg, Russia, July 4-8

Singh M, Naughton J, Yamashita T, Nagai H, Asai K (2011) Surface pressure and flow field behind an oscillating fence submerged in turbulent boundary layer. Exp Fluids 50:701-714

Spazzini P, Iuso G, Onorato M, Zurlo N, Cicca GD (2001) Unsteady behavior of back-facing step flow. Exp Fluids 30:551-561

Statnikov V, Glatzer C, Meiss JH, Meinke M, Schröder W (2012) Numerical investigation of the near wake of generic space launcher systems at transonic and supersonic flows. In: EUCASS Flight Physics Book, vol 5

Sugimoto T, Kitashima S, Numata D, Nagai H, Asai K (2012) Characterization of frequency response of pressure-sensitive paints. Nashville, Tennessee, USA, 50th AIAA Aerospace Sciences Meeting, AIAA Paper 2012-1185

Woodmansee MA, Dutton JC (1998) Treating temperature-sensitivity effects of pressure-sensitive paint measurements. Exp Fluids 24:163-174

Yang L, Zare-Behtash H, Erdem E, Kontis K (2012) Application of aa-psp to hypersonic flows: The double ramp model. Sens Actuators B Chem 161(1):100-107 